\title{
Chronic Early-life Stress in Rat Pups Alters Basal Corticosterone, Intestinal Permeability, and Fecal Microbiota at Weaning: Influence of Sex
}

\author{
Nabila Moussaoui, ${ }^{*}$ Jonathan P Jacobs, Muriel Larauche, Mandy Biraud, Mulugeta Million, Emeran Mayer, and Yvette Taché \\ G. Oppenheimer Center for Neurobiology of Stress and Resilience, CURE: Digestive Diseases Research Center, Vatche and Tamar Manoukian \\ Division of Digestive Diseases, Department of Medicine and Brain Research Institute, University of California, and VA Greater Los Angeles \\ Healthcare System, Los Angeles, California, USA
}

\begin{abstract}
Background/Aims
Wistar rat dams exposed to limited nesting stress (LNS) from post-natal days (PND) 2 to 10 display erratic maternal behavior, and their pups show delayed maturation of the hypothalamic-pituitary-adrenal axis and impaired epithelial barrier at PND10 and a visceral hypersensitivity at adulthood. Little is known about the impact of early life stress on the offspring before adulthood and the influence of sex. We investigated whether male and female rats previously exposed to LNS displays at weaning altered corticosterone, intestinal permeability, and microbiota.
\end{abstract}

\section{Methods}

Wistar rat dams and litters were maintained from PND2 to 10 with limited nesting/bedding materials and thereafter reverted to normal housing up to weaning (PND21). Control litters had normal housing. At weaning, we monitored body weight, corticosterone plasma levels (enzyme immunoassay), in vivo intestinal to colon permeability (fluorescein isothiocyanate-dextran $4 \mathrm{kDa}$ ) and fecal microbiota (DNA extraction and amplification of the $\mathrm{V} 4$ region of the $16 \mathrm{~S}$ ribosomal RNA gene).

\section{Results}

At weaning, LNS pups had hypercorticosteronemia and enhanced intestinal permeability with females $>$ males while body weights were similar. LNS decreased fecal microbial diversity and induced a distinct composition characterized by increased abundance of Gram positive cocci and reduction of fiber-degrading, butyrate-producing, and mucus-resident microbes.

\section{Conclusions}

These data indicate that chronic exposure to LNS during the first week post-natally has sustained effects monitored at weaning including hypercorticosteronemia, a leaky gut, and dysbiosis. These alterations may impact on the susceptibility to develop visceral hypersensitivity in adult rats and have relevance to the development of irritable bowel syndrome in childhood.

(J Neurogastroenterol Motil 2017;23:135-143)

Key Words

Corticosterone; Microbiota; Permeability; Stress, psychological; Weaning

Received: June 26, 2016 Revised: August 9, 2016 Accepted: October 11, 2016

(a) This is an Open Access article distributed under the terms of the Creative Commons Attribution Non-Commercial License (http://creativecommons. org/licenses/by-nc/4.0) which permits unrestricted non-commercial use, distribution, and reproduction in any medium, provided the original work is properly cited.

*Correspondence: Nabila Moussaoui, PhD

CURE: VA GLAHS, Bldg. 115, Rm. 111, 1301 Wilshire Blvd., Los Angeles, CA 90073, USA

Tel: +1-310-478-3711 , Fax: +1-310-268-4963, E-mail: NMoussaoui@mednet.ucla.edu

Nabila Moussaoui and Jonathan P Jacobs contributed equally to this work. 


\section{Introduction}

The impact of early-life stress events on the development of psychiatric illnesses and gastrointestinal disorders such as irritable bowel syndrome (IBS) is well recognized in humans. ${ }^{1,2}$ In preclinical studies, rodents raised under conditions of intermittent maternal separation develop features of IBS-like manifestations and alterations in behavior when they are adults. ${ }^{3-6}$ Another model of early-life adverse events is the rearing of pups by dams subjected postpartum to conditions of limited bedding and nesting stress. ${ }^{7,8}$ This model has face validity in humans ${ }^{7,8}$ where the mother is continuously present but provides an altered repertoire of maternal behaviors indicative of fragmented and erratic care ${ }^{9}$ and altered dam-pup interactions. ${ }^{10}$ It has also the advantage of minimizing variability linked to handling of the pups to perform daily maternal separation., ${ }^{8,11}$ Disturbance of maternal behavior by limited nesting stress (LNS) exposure during the post-natal days (PND) 2 to 10 impacts significantly on the development of the hypothalamic-pituitary-adrenal (HPA) axis in offspring with contrasting effects on corticosteronemia according to the strain studied. Indeed, Sprague-Dawley pups showed either no change ${ }^{7,12}$ or increased ${ }^{13}$ levels at PND9, whereas PND10 Wistar pups exhibited a delayed maturation of the adrenal glands with significant reduction in plasma corticosterone. ${ }^{14}$ PND10 Wistar pups also showed elevated intestinal barrier permeability selectively in males. ${ }^{14}$

At weaning, the HPA axis is still in development ${ }^{15,16}$ and the intestinal barrier permeability to macromolecules is high and declines thereafter although it remains more permeable up to PND40. ${ }^{17-19}$ During this period, bacterial colonization is in process and promotes the development of food tolerance and immune function. ${ }^{20}$ Of importance, recent reports in rodents indicate that early-life stress may influence the microbiota which could be associated with long lasting effects. ${ }^{21-23}$ In particular, maternal separation for 3 hours per day from PND2-9 results in a distinct fecal microbiota in adult rats. ${ }^{22}$ However, whether early life adverse event is associated with alterations of gut microbiota diversity and community structure during development is yet to be established.

In the present study, we investigated whether chronic post-natal stress induced by exposure to LNS from PND2 to 10 influences basal corticosterone and in vivo intestinal-colonic permeability at weaning after 11 days of normal housing. To assess the specificity of the changes induced by LNS, we also included a group of offspring subjected to a brief maternal separation (BMS) for 15 minutes per day from PND2-9 and similarly assessed plasma corticosterone levels and gut permeability at weaning. This BMS procedure is known to improve resilience and HPA axis response to stress in adulthood. ${ }^{24,25}$ Lastly, we examined changes in fecal microbiota of LNS offspring at weaning using high-throughput 16S rRNA taxonomic profiling. Based on recent reports showing that LNS from PND2-9 impacts on the visceral hypersensitivity in a sex-dependent manner in adult rats, ${ }^{26,27}$ the study included pups of both sexes to evaluate whether sexual dimorphism was maintained at weaning as we reported at PND10. ${ }^{14}$

\section{Materials and Methods}

\section{Animals}

Primiparous pregnant Wistar rats obtained on embryonic day E-15 from Harlan facilities (San Diego, California, CA, USA) were housed individually in polypropylene cages under conditions of controlled temperature $\left(23 \pm 1^{\circ} \mathrm{C}\right)$ and lighting $(12: 12$ hour light/dark cycle — 6 PM lights off/6 AM lights on). Food (Prolab RMH 2500; PMI Nutrition International, Inc, Brentwood, MO, USA) and water were available ad libitum. Bedding was changed 3 days before the expected parturition. Cages were inspected for the presence of pups at a 12-hour interval and the date of birth was labeled as day 0 . On PND2, between 9 AM and $10 \mathrm{AM}$, pups were sexed and litters were mixed and culled to a maximum of 12 pups and minimum of 10 with an equal number of each sex as in our previous study. ${ }^{14}$ All experimental protocols (\#09026-11) were approved by the IACUC Committee of the VA Greater Los Angeles Healthcare System which is under the auspices of the OLAW Assurance of Compliance (A3002-01) in accordance with NIH guidelines.

\section{Limited Nesting Stress}

The LNS conditions were similar to those originally described by Gilles et $\mathrm{al}^{7}$ and detailed in our previous studies. ${ }^{14}$ Briefly, on PND2, each mother with pups was placed in a standard cage fitted with a wire mesh bottom (McNichols Quality Standard Expanded Metal, Aluminum Type 3003-H14, 3/16 .032 Standard; McNichols $\mathrm{Co}$, Los Angeles, CA, USA) that was positioned $2.5 \mathrm{~cm}$ above the floor to allow passage of excrements. The bedding was reduced to cover the cage floor sparsely and nesting material consisted of half a piece of paper towel $(10 \times 5 \mathrm{~cm})$ that the dam shred and used to construct a rudimentary nest area. Control groups (CTL) were maintained in standard cages with direct contact with standard amount of bedding, and one paper towel as nesting material. 
Thereafter, dams and pups were left completely undisturbed. Cages were unchanged from PND2 to 10 and located in a separate room dedicated to this study. At PND10, pups and dams were returned to normal bedding until PND21 when parameters were monitored.

\section{Brief Maternal Separation}

The BMS was performed as described previously. ${ }^{14,25}$ Housing conditions were similar to those of the CTL group except that from PND2 to 9, pups were separated from the dam for 15 minutes daily starting between $9 \mathrm{AM}$ and $10 \mathrm{AM}$. For each litter, pups were removed all together from their home cage and kept in temperature controlled cages at $28 \pm 1^{\circ} \mathrm{C}$ with normal bedding.

\section{Blood and Fecal Sampling at Post-natal Day 21}

PND21 male and female pups from CTL and LNS litters (2 each) were weighed and in one litter of each group, PND21 male and female pups were euthanized by decapitation and the adrenal glands were harvested and weighed. In all pups, blood sample was collected from the facial vein at $1 \mathrm{PM}$ in lightly hand restrained conditions for corticosterone plasma determination and intestinal permeability. The time of blood collection was dictated by the 4-h permeability assay. Feces were collected after the permeability assay from CTL and LNS litter (one litter each) at PND21 and stored at $-80^{\circ} \mathrm{C}$ until microbiota analysis.

\section{Plasma Corticosterone Determination}

Blood samples were collected from the facial vein in lightly restrained conscious rats with a collection time less than 30 seconds as performed in our previous study. ${ }^{147}$ Samples were centrifuged (10 minutes, $2500 \mathrm{~g}$ ) and plasma was stored at $-80^{\circ} \mathrm{C}$ until assay. Plasma corticosterone concentration was determined by enzyme immunoassay (Cayman Chemical, Ann Arbor, MI, USA) according to the manufacturer's instructions. The sensitivity of the assay was $150 \mathrm{pg} / \mathrm{mL}$ and interassay variability was $11 \%$.

\section{In Vivo Intestinal Permeability}

Total (small and large intestine) permeability was measured in vivo as detailed in our previous studies. ${ }^{14,17}$ Briefly, at 9 AM pups received oral gavage $(70 \mu \mathrm{L})$ of fluorescein isothiocyanate (FITC) labeled 4 kDa dextran (FD4) (4 kDa; Sigma, Milwaukee, WI, USA), at the dose of $750 \mathrm{mg} / \mathrm{kg}$ body weight in $0.9 \% \mathrm{NaCl}$. After 4 hours, a sufficient transit time for FD4 to be recovered into the colon lumen, ${ }^{17}$ rats were lightly hand restrained and blood (200 $\mu \mathrm{L}$ ) was withdrawn from the facial vein using heparin-coated capillaries (SARSTEDT, Saint James Church Road, NC, USA).
Samples were placed immediately on ice and centrifuged. Intestinal permeability to FD4 was determined by measuring plasma FD4 concentration using an automatic Synergy HT multi-detection microplate reader (Ex 485 nm and Em 525 nm; BioTek, Winooski, VT, USA).

\section{Microbiota Analysis}

Feces were collected from PND21 male and female rats for DNA extraction and amplification of the V4 region of the 16S rRNA gene. Genomic DNA was extracted using the Powersoil kit as per manufacturer's instructions (MoBio Laboratories Inc, Carlsbad, CA, USA). The V4 region of $16 \mathrm{~S}$ ribosomal RNA genes was amplified and underwent paired end sequencing on an Illumina MiSeq using the $2 \times 150 \mathrm{bp} v 2$ kit as previously described. ${ }^{28}$ The 253 base pair reads were processed using QIIME v1.9.1 with default parameters. ${ }^{29}$ Observable taxonomic units (OTUs) were picked against the May 2013 version of the Greengenes database, pre-filtered at 97\% identity. Sequence depth ranged from 75603 to 110767 . Alpha diversity metrics (ie, bacterial diversity within a sample) and beta diversity (differences in composition across samples) were calculated in QIIME v1.9.1 using OTU-level data rarefied to 75603 sequences. Alpha diversity metrics included Faith's phylogenetic diversity metric, Chaol, and Shannon index.

\section{Statistical Methods}

Data are expressed as mean \pm SEM and were analyzed using GraphPad Prism 4 software (GraphPad, San Diego, CA, USA). Plasma corticosterone in CTL and LNS groups were compared by Student's $t$ test. Pearson's correlation coefficient was used to assess the correlation between corticosterone plasma levels and adrenals weights/100 g body weight and between corticosterone plasma levels and Akkermansia abundance. Correlation analysis between corticosteronemia and adrenal weights were only reported in female rats as no significant change in adrenal weights was observed between male pups exposed or not to LNS procedure. One-way ANOVA followed by Tukey post hoc test comparisons were used to analyze in vivo intestinal permeability. Interaction between treatment and sex was analyzed by two-way ANOVA followed by Bonferroni post hoc test. A $P$-value $<0.05$ was considered significant. The significance of differences in alpha diversity was calculated using the Mann-Whitney U test. Beta diversity was calculated using unweighted UniFrac and visualized by principal coordinates analysis. Adonis, a permutational analysis of variance, was performed using 100000 permutations to test for differences in beta diversity between the LNS and control groups. ${ }^{30}$ Association of microbial 
genera and OTUs with LNS or control groups were evaluated using DESeq2 in R. ${ }^{31}$ Unrarefied $16 \mathrm{~S}$ rRNA count data was first filtered to remove OTUs present in only one sample then fitted to multivariate negative binomial models with LNS group and sex as covariates. $P$-values for differential abundance were converted to q-values to correct for multiple hypothesis testing $(P<0.05$ for significance). ${ }^{32}$ DESeq2 models were also run with intestinal permeability or plasma corticosterone as covariates in addition to LNS group and sex to identify taxa associated with these parameters.

\section{Results}

\section{Limited Nesting Stress from Post-natal Days 2-10 Did Not Alter Pups Body Weight at Weaning}

No significant difference was observed in body weights between PND21 male and female Wistar rats in the CTL group (36.4 $\pm 1.0 \mathrm{~g}, \mathrm{n}=9$ vs $35.3 \pm 1.2 \mathrm{~g}, \mathrm{n}=14$, respectively, $P=0.500$ ). Body weights in the LNS group did not differ significantly when compared to the same sex CTL group (males: LNS $34.7 \pm 0.4 \mathrm{~g}$ vs control $36.4 \pm 1.0 \mathrm{~g}, P=0.100, \mathrm{n}=9-12$; females: LNS 33.4 $\pm 0.4 \mathrm{~g}$ vs control $35.3 \pm 1.2 \mathrm{~g}, P=0.200, \mathrm{n}=11-14)$. There was no significant sex difference in the body weight of the LNS group (two-way ANOVA: F $[1,40]=1.8, P=0.200$ ).

\section{Limited Nesting Stress from Post-natal Days 2-10 Increased Plasma Corticosterone Levels in Pups at Weaning}

In PND21 males, plasma corticosterone values showed a trend to be higher in LNS group compared to CTL which did not reach statistical significance $(4.0 \pm 0.4 \mu \mathrm{g} / \mathrm{dL}$ vs $2.4 \pm 0.7 \mu \mathrm{g} / \mathrm{dL}, P=$ $0.050, n=8-9)$ (Fig. 1A). By contrast, corticosterone plasma levels in the LNS female group increased significantly by $147 \%$ in comparison to CTL female pups $(9.4 \pm 1.6 \mu \mathrm{g} / \mathrm{dL}$ vs $3.8 \pm 0.9 \mu \mathrm{g} /$ $\mathrm{dL}, P<0.01, \mathrm{n}=6-12$ ) (Fig. 1A). Two-way ANOVA showed highly significant differences by treatment group $(\mathrm{F}[2,40]=10.5$, $P<0.001)$ and $\operatorname{sex}(\mathrm{F}[1,40]=12, P<0.01)$.

PND21 LNS females displayed a significant increase in adrenal weight ( $\mathrm{mg} / 100 \mathrm{~g}$ body weight) in comparison to the CTL group (LNS $35.1 \pm 0.7$ vs control $30.0 \pm 2.0, P<0.05, \mathrm{n}=4$ ) whereas LNS male did not show significant changes compared to the male CTL and had rather a tendency to be lower than the CTL (LNS $29.0 \pm 1.1$ vs control 34.6 $\pm 3.0, P=0.200, \mathrm{n}=3-4$ ). Positive correlation between corticosterone plasma levels and the adrenal weights ( $\mathrm{mg} / 100 \mathrm{~g}$ body weight) was observed in female CTL and LNS pups (Pearson $r=0.75, P<0.05, \mathrm{n}=8$ ) (Fig. $1 \mathrm{~B})$, but not in males (not shown).

By contrast, PND21 BMS pups, either male or female, did not show significant changes in plasma corticosterone levels (BMS male: $2.0 \pm 0.4$ vs controls $2.4 \pm 0.7 \mu \mathrm{g} / \mathrm{dL}, P=0.300, \mathrm{n}=4-8$; BMS female: $3.5 \pm 0.8$ vs controls $3.8 \pm 0.9 \mu \mathrm{g} / \mathrm{dL}, P=0.200$, $\mathrm{n}=7-12)$.

\section{Limited Nesting Stress from Post-natal Days 2-10 Increased In Vivo Intestinal Permeability in Females at Weaning}

Intestinal permeability to FD4 was not significantly different between male and female pups in the CTL group $(\mu \mathrm{g} / \mathrm{mL}$ plasma: $8.9 \pm 0.6$ vs $9.4 \pm 0.7$, respectively). Limited nesting stress increased intestinal permeability to FD4 by $70 \%$ in female pups $(P$
A

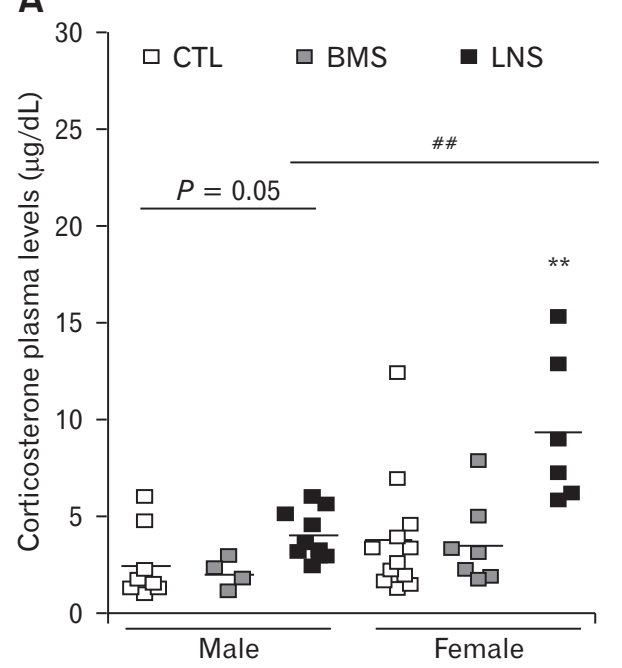

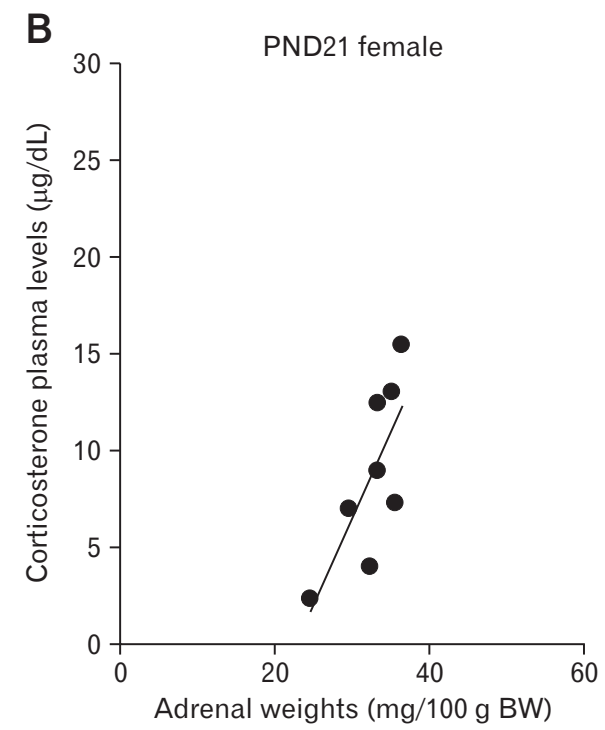

Figure 1. Limited nesting/bedding stress from post-natal days (PND) 2 to 10 increases corticosterone plasma levels in PND21 pups. (A) Corticosterone plasma levels in male and female PND21 pups in limited nesting stress (LNS), brief maternal separation (BMS), and control (CTL) groups. Data are mean \pm SEM (4-12 animals per group). ${ }^{*} P<0.01 \mathrm{LNS}$ female vs CTL female pups, ${ }^{\# \#} P<0.01 \mathrm{LNS}$ male vs LNS female pups. (B) Positive correlation (Pearson $r=0.75, P=0.05$, $\mathrm{n}=8$ ) between corticosterone plasma levels and adrenals weights/100 g body weight (BW) in female PND21 pups from LNS and CTL groups. 


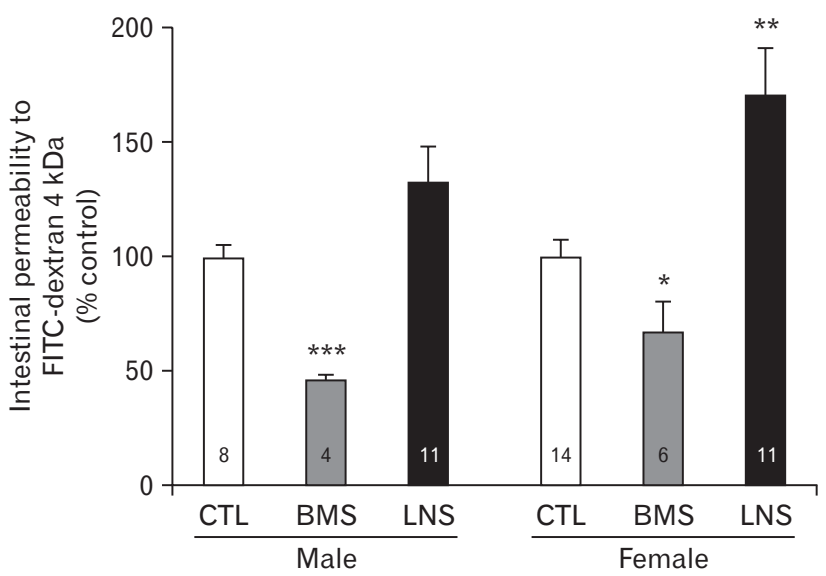

Figure 2. Intestinal permeability to fluorescein isothiocyanate (FITC) labeled $4 \mathrm{kDa}$ dextran (FD4) is increased in post-natal days (PND) 21 pups exposed to limited nesting/bedding stress from PND2-10. In vivo intestinal permeability to FD4 in male and female PND21 pups in limited nesting stress (LNS), brief maternal separation (BMS), and control (CTL) groups. Data are expressed as the mean \pm SEM. ${ }^{*} P<0.05,{ }^{* *} P<0.01,{ }^{* *} P<0.001$ compared to corresponding control group.

$<0.01$ ) while inducing a $32 \%$ elevation in male pups that did not reach significance. In contrast, pups exposed to BMS showed a significant 54\% $(P<0.001)$, and 33\% $(P<0.05)$ decrease of intestinal permeability in males and females respectively compared to the CTL group (Fig. 2).

Two-way ANOVA revealed significant effect of treatment $(\mathrm{F}$ $[2,48]=22, P<0.0001)$ and $\operatorname{sex}(\mathrm{F}[1,48]=4.8, P<0.05)$.

\section{Limited Nesting Stress from Post-natal Days 2-10 Induced a Lower Diversity in Fecal Microbiota and Increased Abundance of Genera of Gram Positive Cocci at Weaning}

The LNS group had decreased fecal microbial diversity using 3 metrics: Chaol $(P=0.0003)$, phylogenetic diversity $(P=0.0005)$, and Shannon index $(P=0.004)$ (Fig. 3A). Beta diversity analysis using unweighted UniFrac demonstrated that the LNS group had a distinct fecal microbiota composition compared to CTL $(P=$ 0.00009) (Fig. 3B). Differential abundance testing was performed using DESeq2 to identify microbes associated with LNS after adjusting for sex. The LNS group had decreased abundance of 24 genera (representing 487 OTUs) at a significance threshold of $\mathrm{q}$ $<0.05$ (Fig. 3C). This included fiber-degrading microbes such as Oscillospira (47 OTUs), Ruminococcus (17), and Lachnospira (3); butyrate-producing microbes such as Roseburia (27), Coprococcus (10), and Eubacterium dolichum; and the mucus-resident bacteria Akkermansia mucinophila and Mucispirillum schaedleri. There was increased abundance of 20 genera (representing 204 enriched OTUs), of which 8 were Gram positive cocci: Enterococcus (6 OTUs), unclassified Enterococcaceae (2), Streptococcus (4), unclassified Streptococcaceae (1), Peptococcus (2), Aerococcus (3), Jeotgalicoccus (2), and Facklamia (1). Additional enriched genera included Clostridium (4), Corynebacterium (4), Desulfovibrio (1), Granulicatella (2), Rothia (1), and Proteus (1). No genera and only 2 OTUs were significantly associated with sex.

\section{Correlation Analyses Between Corticosterone Plasma Level, Intestinal Permeability, and Microbiota}

No correlation was observed between corticosterone plasma levels and intestinal permeability values in both sexes (Pearson $r=$ $-0.16, P=0.300, \mathrm{n}=36$ ) or in each sex analyzed separately (data not shown). The association of the intestinal microbes with corticosterone and intestinal permeability was then assessed using a multivariate approach adjusting for LNS group and sex. One genus, Akkermansia, had a statistically significant negative association with plasma corticosterone $(q=0.02)$. Log transformed abundances of Akkermansia were negatively correlated with plasma corticosterone with Pearson's $r=-0.82(P=0.0001)$ (Fig. 3D). No taxa were associated with intestinal permeability after adjusting for LNS group and sex.

\section{Discussion}

The present study indicates that exposure of Wistar pups to LNS post-natally from days 2 to 10 induced alteration in plasma corticosterone levels, gut permeability, and fecal microbiota as monitored 11 days after the end of LNS at the time of weaning.

We reported previously that PND10 Wistar pups exposed to LNS from PND2 to 10 had delayed maturation of the HPA axis as shown by a significant reduction of corticosterone plasma levels and adrenal glands weight/100 g body weight in both males and females. ${ }^{14}$ In the present study, PND21 male and female pups exposed to similar conditions of LNS followed by 11 days of normal housing showed 67\% $(P=0.050)$ and $147 \%(P<0.01)$ higher basal corticosterone plasma levels respectively than same sex controls with values in females being significantly superior than in males. Exposure to early-life stress events has been reported to have a long lasting effect on HPA axis activity in adult rodents previously exposed to repeated maternal separation ${ }^{22}$ and in adult humans. ${ }^{33,34}$ The present results indicate that rat pups exposed to the LNS post-natal stress associated with altered maternal behavior ${ }^{9,12,14}$ induces a basal state of elevated corticosteronemia which can be detected at weaning more prominently in females while not present 
A



C

\section{Methanosphaera-
Proteus- \\ Turicibacter-}

Enterococcaceae (f)

Streptococcaceae (f)
Corynebacterium

Enterococcus -

Peptococcus

Facklamia -

Lactococcus

Desulfovibrio

Granulicatella -

Aerococcus

Coprobacillus -

Streptococcus

Clostridium

Rothia

Clostridiaceae (f)

Holdemenia-

Ruminococcus -

Ruminococcaceae (f)

[Barnesiellaceae] (f)

Staphylococcus

Oscillospira

Parabacteroides Mucispirillum

Peptococcaceae (f)

Erysipelotrichaceae (f)

Clostridiales (o)

Coprococcus

Dehalobacterium

Akkermansia-

Lachnospira

Anaerotruncus -

Haemophilus

RF32 (o)

Odoribacter

Victivallaceae (f)

RF39 (o)

Roseburia

YS2 (o)

Anaeroplasma
[Eubacterium]

\section{Abundance}

- $<0.0001$

- 0.0005

- 0.005

- 0.02

0.07



Phylum

- Other
B

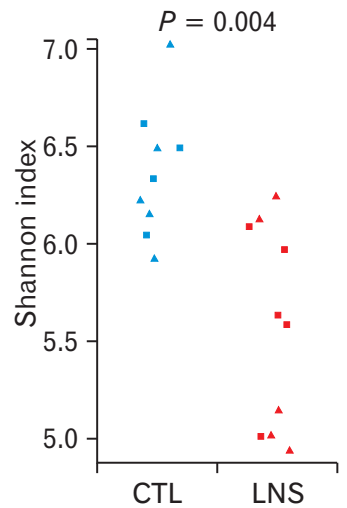

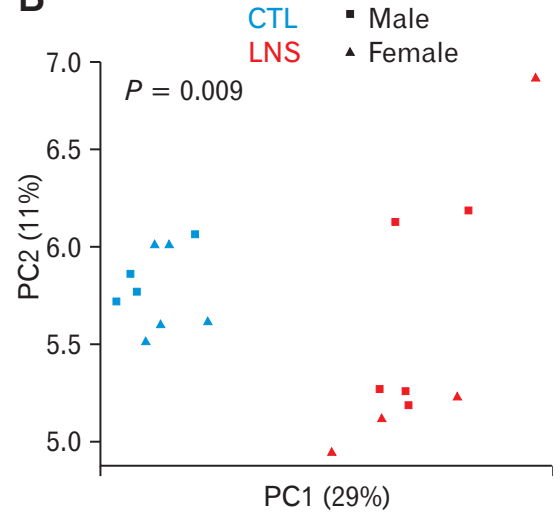

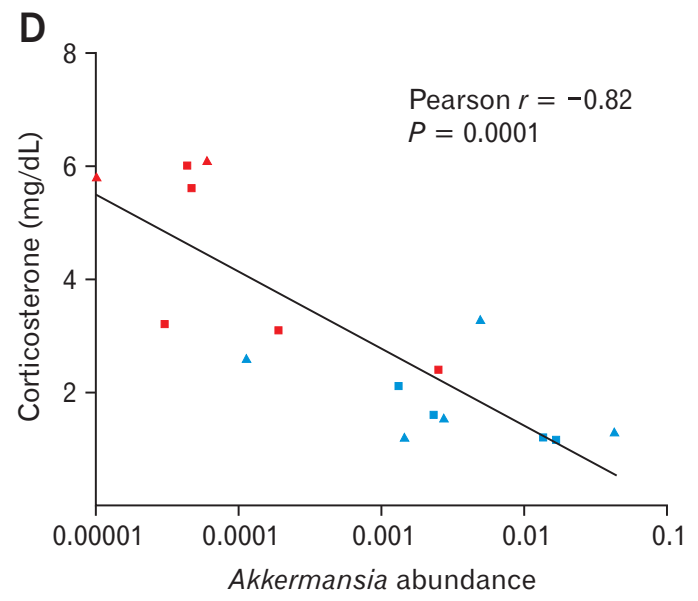

Akkermansia abundance

- Bacteroidetes

- Firmicutes

- Proteobacteria



Figure 3. Rats exposed to limited nesting/bedding stress have a less diverse fecal microbiota characterized by expansion of microbes such as Staphylococcus, Proteus, and Enterococcus and depletion of short chain fatty acid producing organisms such as Roseburia and Coprococcus. (A) Three measures of microbial diversity, color-coded by limited nesting stress (LNS) group. Squares represent males and triangles represent females. $P$-values were calculated using the Mann-Whitney U test. (B) Principal coordinates analysis plot colored by LNS group. $P$-value for the difference in microbial composition between groups was calculated using Adonis. (C) Genera with differential abundance in the LNS group compared to the control (CTL) group are shown, ordered by magnitude of effect and color-coded by phylum. Circle size represents the mean normalized relative abundance of the genus across all the samples. Log2 fold change was calculated using multivariate models incorporating sex and LNS. (D) Plasma corticosterone levels were correlated with relative abundance of Akkermansia on a log scale. Color represents LNS group and symbols represent sex. 
at PND10. ${ }^{14}$ This shows for the first time the onset of sex differences in a component of the HPA axis induced by early-life adverse environmental factors. The hypercorticosteronemia is specific to the chronic stress exposure from PND2-10 as BMS during the same period did not influence basal plasma corticosterone levels either at PND10 11 or at PND21 (present study) in both male and female pups.

The LNS model enables to study the impact of chronic postnatal stress on the trajectory of developing intestinal barrier permeability. LNS exposure from PND2-10 is associated with increased total intestinal permeability to FD4 predominantly in PND21 female pups, even so, pups were subsequently housed for 11 days under normal nesting/bedding conditions. By contrast, we described previously that PND10 pups exposed similarly to LNS exhibited a significant increase of intestinal permeability occurring mainly in male pups. ${ }^{14}$ The temporal sex differences observed here could not be explained by hormonal actions as efficient secretion of sexual hormones does not occur until PND30 in rats. ${ }^{35}$ Collectively, these findings point to a sex difference in developmental susceptibility to early-life stress with immediate and long lasting alterations in intestinal barrier function occurring later in females compared to males. Other studies performed in male rats indicate an alteration of the intestinal barrier at weaning in the maternal separation model ${ }^{23}$ that was detected by the significant increase of colonic short-circuit current and horseradish peroxidase flux along with increased intestinal permeability that was still maintained in adulthood. ${ }^{6,34}$ Glucocorticoids play a role in the increased intestinal permeability induced by a short acute maternal separation stress in PND10 pups $^{17}$ but not in adult rats subjected to repeated maternal separation. ${ }^{36,37} \mathrm{In}$ the present study, we found no correlation between corticosterone plasma levels and intestinal permeability at weaning, either in LNS or BMS models, suggesting that glucocorticoids are not involved in the mechanisms contributing to the altered permeability. Peripheral corticotropin-releasing factor receptors and nerve growth factors have been identified as major underlying mechanisms of the enhanced intestinal permeability in adult rats exposed to intermittent maternal separation post-natally. ${ }^{36,37}$ Whether similar mechanisms are implicated in the increased permeability during the developmental period at weaning need to be further investigated. Intestinal barrier disruption has been described to occur in IBS patients, ${ }^{38-41}$ although the link between early adverse life events and increased intestinal permeability needs to be evaluated based on the present experimental data.

On the other hand, BMS exposure is associated with a significant lower intestinal permeability than the CTL group at weaning in both sexes which was already observed at PND10. ${ }^{14}$ Together these findings are indicative that short maternal separation accelerates maturation of intestinal barrier function during development stages in both male and female pups. Considering the role of alterations in intestinal permeability in stress-sensitive IBS manifestation, ${ }^{42}$ the present data may have a bearing with our previous report showing that exposure to BMS reduced the visceral pain to colorectal distension monitored 1 hour after exposure to water avoidance stress and prevents the hyperalgesia 24 hours later, while repeated 3-hour maternal separations resulted in a hyperalgesic response when tested in adulthood. ${ }^{43}$

There is growing recognition that the neonatal intestinal microbiota plays a critical role in neurodevelopment and postnatal HPA stress response. ${ }^{44}$ Recent evidence also indicates that the gut microbiota contributes to the expression of behavioral alterations in adult mice exposed to early maternal separation. ${ }^{21}$ The present study indicates that PND21 rats reared in LNS conditions have decreased fecal microbiota diversity and a distinct composition compared to CTL rats. LNS exposure results in a decrease abundance of fiberdegrading, butyrate-producing, and mucus-resident bacteria compared with controls. These bacteria play an important role in the maintenance of the intestinal barrier. ${ }^{45}$ We also found that feces of LNS rats had decreased abundance of 19 OTUs belonging to the Lactobacillus genus, paralleling results in one study showing the reduction of Lactobacillus species in the distal colon of PND20 rats exposed to maternal separation from PND4-19, although no other taxa were investigated. ${ }^{23}$ We observed in LNS rats an increased abundance of Gram positive cocci such as Enterococcus, Enterococcaceae, and Streptococcus which were reported to be dominant bacteria in fecal microbiota of PND14 rats. ${ }^{46}$ Those data suggest that LNS rats may have delayed maturation of the neonatal microbiota. Of note, no significant sex difference was observed in either the diversity or composition of fecal microbiota in weaned rats, and microbial changes did not correlate with the degree of intestinal permeability after adjustment for the effect of LNS. However, the analysis of the microbiota was performed in feces and not in the intestine, and we could not exclude the possibility that alterations in bacteria adhering to the gut mucosa could potentially be involved in the modulation of the HPA axis and the intestinal permeability induced by LNS.

Sustained perturbations in the intestinal microbiota related to early-life stress may be a potential contributing mechanism by which early adverse life events can be linked to the susceptibility of developing IBS later in life, given the extensive literature suggesting a role of the microbiota in the pathogenesis of IBS. ${ }^{47,48}$ Recent studies showed rats exposed to LNS from PND 2-9 display visceral hypersensitivity when they are adults. ${ }^{26,27}$ Disturbance of bacterial 
colonization by antibiotic treatment from PND4 to 13 induces visceral hypersensitivity in adult rats which is more pronounced in males. ${ }^{49}$ Conversely, probiotic treatment from PND3 to 60 has a protective effect on the development of visceral hyperalgesia in $\mathrm{Wi}$ star rats exposed to maternal separation stress for 3 hours per day from PND2 to $14 .^{50}$ Taken together, the present and previous studies point to a potential important role played by dysbiosis during the early-life period in modulating the visceral sensitivity of the host.

In conclusion, the present study shows for the first time that chronic early exposure of dams to LNS for the PND2-10 period impacts the offspring development during the critical suckling period. PND21 rats had an elevated basal level of circulating corticosterone and increased in vivo intestinal permeability more prominently in females. By contrast, BMS exposure was associated with a significant decrease of intestinal permeability to FD4 without changes in corticosterone plasma levels compared to the control group. Moreover, the study provides the first evaluation of the fecal microbiota diversity and composition in weaning rats exposed to chronic stress during the first post-natal week. LNS rats display lower bacteria diversity with a decrease in fiber-digesting, mucus resident and butyrate-producing bacteria, while other bacteria including multiple genera of Gram positive cocci were more abundant. The time of weaning is an important development time and our findings may have implications in understanding the impact of early stress on the onset of IBS in children. ${ }^{51}$ Moreover, alterations induced by LNS may have relevance to the long-lasting consequences of early adverse life events reported in IBS, alterations of HPA axis and its prevalence in women. ${ }^{2,52}$

Acknowledgements: We acknowledge Dr Patrick Frost (Department of Hematology-Oncology, UCLA) for the plate reader use.

Financial support: This work was supported by National Institute of Health grants P50 DK-64539 and Center grant DK-41301 (Animal Model Core), R01 DK-57238, K01 DK088937, and VA Career Scientist Award.

\section{Conflicts of interest: None.}

Author contributions: Nabila Moussaoui, Jonathan P Jacobs, Muriel Larauche, Mulugeta Million, Emeran Mayer, and Yvette Taché conceived and designed the experiments; Nabila Moussaoui, Jonathan P Jacobs, Muriel Larauche, and Mandy Biraud performed the experiments; and Nabila Moussaoui, Jonathan P Jacobs, and Yvette Taché analyzed the data and wrote the paper.

\section{References}

1. Larkin W, Read J. Childhood trauma and psychosis: evidence, pathways, and implications. J Postgrad Med 2008;54:287-293.

2. Bradford K, Shih W, Videlock EJ, et al. Association between early adverse life events and irritable bowel syndrome. Clin Gastroenterol Hepatol 2012;10:385-390.e3.

3. Li M, Xue X, Shao S, Shao F, Wang W. Cognitive, emotional and neurochemical effects of repeated maternal separation in adolescent rats. Brain Res 2013;1518:82-90.

4. O'Leary OF, Cryan JF. Towards translational rodent models of depression. Cell Tissue Res 2013;354:141-153.

5. Barreau F, Ferrier L, Fioramonti J, Bueno L. New insights in the etiology and pathophysiology of irritable bowel syndrome: Contribution of neonatal stress models. Pediatr Res 2007;62:240-245.

6. Gareau MG, Silva MA, Perdue MH. Pathophysiological mechanisms of stress-induced intestinal damage. Curr Mol Med 2008;8:274-281.

7. Gilles EE, Schultz L, Baram TZ. Abnormal corticosterone regulation in an immature rat model of continuous chronic stress. Pediatr Neurol 1996;15:114-119.

8. Molet J, Maras PM, Avishai-Eliner S, Baram TZ. Naturalistic rodent models of chronic early-life stress. Dev Psychobiol 2014;56:1675-1688.

9. Ivy AS, Brunson KL, Sandman C, Baram TZ. Dysfunctional nurturing behavior in rat dams with limited access to nesting material: a clinically relevant model for early-life stress. Neuroscience 2008;154:1132-1142.

10. Heun-Johnson H, Levitt P. Early-life stress paradigm transiently alters maternal behavior, dam-pup interactions, and offspring vocalizations in mice. Front Behav Neurosci 2016;10:142.

11. Sorge RE, Martin LJ, Isbester KA, et al. Olfactory exposure to males, including men, causes stress and related analgesia in rodents. Nat Methods 2014;11:629-632.

12. McLaughlin RJ, Verlezza S, Gray JM, Hill MN, Walker CD. Inhibition of anandamide hydrolysis dampens the neuroendocrine response to stress in neonatal rats subjected to suboptimal rearing conditions. Stress 2016;19:114-124.

13. Avishai-Eliner S, Gilles EE, Eghbal-Ahmadi M, Bar-El Y, Baram TZ. Altered regulation of gene and protein expression of hypothalamicpituitary-adrenal axis components in an immature rat model of chronic stress. J Neuroendocrinol 2001;13:799-807.

14. Moussaoui N, Larauche M, Biraud M, et al. Limited nesting stress alters maternal behavior and in vivo intestinal permeability in male Wistar pup rats. PLoS one 2016;11:e0155037.

15. Schmidt M, Enthoven L, van Woezik JHG, Levine S, de Kloet ER, Oitzl MS. The dynamics of the hypothalamic-pituitary-adrenal axis during maternal deprivation. J Neuroendocrinol 2004;16:52-57.

16. Vázquez DM. Stress and the developing limbic-hypothalamic-pituitaryadrenal axis. Psychoneuroendocrinology 1998;23:663-700.

17. Moussaoui N, Braniste V, Ait-Belgnaoui A, et al. Changes in intestinal glucocorticoid sensitivity in early life shape the risk of epithelial barrier defect in maternal-deprived rats. PLoS one 2014;9:e88382.

18. Iiboshi Y, Nezu R, Khan J, et al. Developmental changes in distribution 
of the mucous gel layer and intestinal permeability in rat small intestine. JPEN J Parenter Enteral Nutr 1996;20:406-411.

19. Telemo E, Weström BR, Ekström G, Karlsson BW. Intestinal macromolecular transmission in the young rat: influence of protease inhibitors during development. Biol Neonate 1987;52:141-148.

20. Turner JR. Intestinal mucosal barrier function in health and disease. Nat Rev Immunol 2009;9:799-809.

21. De Palma G, Blennerhassett P, Lu J, et al. Microbiota and host determinants of behavioural phenotype in maternally separated mice. Nat Commun 2015;6:7735.

22. O'Mahony SM, Marchesi JR, Scully P, et al. Early life stress alters behavior, immunity, and microbiota in rats: implications for irritable bowel syndrome and psychiatric illnesses. Biol Psychiatry 2009;65:263-267.

23. Gareau MG, Jury J, MacQueen G, Sherman PM, Perdue MH. Probiotic treatment of rat pups normalises corticosterone release and ameliorates colonic dysfunction induced by maternal separation. Gut 2007;56:1522-1528.

24. Lyons DM, Parker KJ, Schatzberg AF. Animal models of early life stress: implications for understanding resilience. Dev Psychobiol 2010;52:616624.

25. McIntosh J, Anisman H, Merali Z. Short- and long-periods of neonatal maternal separation differentially affect anxiety and feeding in adult rats: gender-dependent effects. Brain Res Dev Brain Res 1999;113:97-106.

26. Guo Y, Wang Z, Mayer EA, Holschneider DP. Neonatal stress from limited bedding elicits visceral hyperalgesia in adult rats. Neuroreport 2015;1:13-16.

27. Prusator DK, Greenwood-Van Meerveld B. Gender specific effects of neonatal limited nesting on viscerosomatic sensitivity and anxiety-like behavior in adult rats. Neurogastroenterol Motil 2015;1:72-81

28. Tong M, Jacobs JP, McHardy IH, Braun J. Sampling of intestinal microbiota and targeted amplification of bacterial $16 \mathrm{~S}$ rRNA genes for microbial ecologic analysis. Curr Protoc Immunol 2014;107:7.41.1-11.

29. Caporaso JG, Kuczynski J, Stombaugh J, et al. QIIME allows analysis of high-throughput community sequencing data. Nat Methods 2010;7:335336.

30. Anderson MJ. A new method for non-parametric multivariate analysis of variance. Austral Ecology 2001;26:32-46.

31. Love MI, Huber W, Anders S. Moderated estimation of fold change and dispersion for RNA-seq data with DESeq2. Genome Biol 2014;15:550.

32. Storey JD, Tibshirani R. Statistical significance for genomewide studies. Proc Natl Acad Sci 2003;100:9440-9445.

33. Heim C, Newport DJ, Mletzko T, Miller AH, Nemeroff CB. The link between childhood trauma and depression: insights from HPA axis studies in humans. Psychoneuroendocrinology 2008;33:693-710.

34. Anacker C, O'Donnell KJ, Meaney MJ. Early life adversity and the epigenetic programming of hypothalamic-pituitary-adrenal function. Dialogues Clin Neurosci 2014;16:321-333.

35. Walker DM, Juenger TE, Gore AC. Developmental profiles of neuroendocrine gene expression in the preoptic area of male rats. Endocrinology 2009;150:2308-2316.

36. Barreau F, Cartier C, Ferrier L, Fioramonti J, Bueno L. Nerve growth factor mediates alterations of colonic sensitivity and mucosal barrier induced by neonatal stress in rats. Gastroenterology 2004;127:524-534.

37. Rodiño-Janeiro BK, Alonso-Cotoner C, Pigrau M, Lobo B, Vicario M, Santos J. Role of corticotropin-releasing factor in gastrointestinal permeability. J Neurogastroenterol Motil 2015;21:33-50.

38. Spiller RC, Jenkins D, Thornley JP, et al. Increased rectal mucosal enteroendocrine cells, $\mathrm{T}$ lymphocytes, and increased gut permeability following acute Campylobacter enteritis and in post-dysenteric irritable bowel syndrome. Gut 2000;47:804-811.

39. Marshall JK, Thabane M, Garg AX, Clark W, Meddings J, Collins $\mathrm{SM}$. Intestinal permeability in patients with irritable bowel syndrome after a waterborne outbreak of acute gastroenteritis in Walkerton, Ontario. Aliment Pharmacol Ther 2004;20:1317-1322.

40. Piche T, Barbara G, Aubert P, et al. Impaired intestinal barrier integrity in the colon of patients with irritable bowel syndrome: involvement of soluble mediators. Gut 2009;58:196-201.

41. Piche T. Tight junctions and IBS - the link between epithelial permeability, low-grade inflammation, and symptom generation? Neurogastroenterol Motil 2014;26:296-302.

42. Zhou Q, Zhang B, Verne GN. Intestinal membrane permeability and hypersensitivity in the irritable bowel syndrome. Pain 2009;146:41-46.

43. Schwetz I, McRoberts JA, Coutinho SV, et al. Corticotropin-releasing factor receptor 1 mediates acute and delayed stress-induced visceral hyperalgesia in maternally separated Long-Evans rats. Am J Physiol Gastrointest Liver Physiol 2005;289:G704-G712.

44. Jašarević E, Howerton CL, Howard CD, Bale TL. Alterations in the vaginal microbiome by maternal stress are associated with metabolic reprogramming of the offspring gut and brain. Endocrinology 2015;156:32653276.

45. Neish AS. Microbes in gastrointestinal health and disease. Gastroenterology 2009;136:65-80.

46. Yajima M, Nakayama M, Hatano S, et al. Bacterial translocation in neonatal rats: the relation between intestinal flora, translocated bacteria, and influence of milk. J Pediatr Gastroenterol Nutr 2001;33:592-601.

47. Simrén M, Barbara G, Flint HJ, et al. Intestinal microbiota in functional bowel disorders: a Rome foundation report. Gut 2013;62:159-176.

48. Barbara G, Feinle-Bisset C, Ghoshal UC, et al. The intestinal microenvironment and functional gastrointestinal disorders. Gastroenterology 2016;150:1305-1318.e8.

49. O'Mahony SM, Felice VD, Nally K, et al. Disturbance of the gut microbiota in early-life selectively affects visceral pain in adulthood without impacting cognitive or anxiety-related behaviors in male rats. Neuroscience 2014;277:885-901.

50. Distrutti E, Cipriani S, Mencarelli A, Renga B, Fiorucci S. Probiotics VSL\#3 protect against development of visceral pain in murine model of irritable bowel syndrome. PLoS One 2013;8:e63893.

51. Chumpitazi BP, Shulman RJ. Underlying molecular and cellular mechanisms in childhood irritable bowel syndrome. Mol Cell Pediatr 2016;3:11.

52. Mulak A, Taché Y, Larauche M. Sex hormones in the modulation of irritable bowel syndrome. World J Gastroenterol 2014;20:2433-2448. 